\title{
A new method for the quantification of aortic calcification by three-dimensional micro-computed tomography
}

\author{
C. HUESA ${ }^{1}$, J.L. MILLÁN ${ }^{2}$, R.J. VAN 'T HOF ${ }^{3}$ and V.E. MacRAE ${ }^{1}$ \\ ${ }^{1}$ The Roslin Institute and Royal (Dick) School of Veterinary Studies, The University of Edinburgh, \\ Midlothian, Scotland, UK; ${ }^{2}$ Sanford Children's Health Research Center, Sanford-Burnham Medical \\ Research Institute, La Jolla, CA, USA; ${ }^{3}$ Rheumatic Diseases Unit, Molecular Medicine Centre, Institute \\ of Genetics and Molecular Medicine, University of Edinburgh, Edinburgh, Scotland, UK
}

Received July 25, 2013; Accepted August 23, 2013

DOI: $10.3892 / \mathrm{ijmm} .2013 .1490$

\begin{abstract}
To gain a better understanding of the mechanisms that underpin aortic calcification, rodent models have been previously utilised. Regions of calcium and phosphate deposition are commonly visualised using labor-intensive two-dimensional histomorphometric techniques. In this study, we developed a novel micro-computed tomography $(\mu \mathrm{CT})$ imaging protocol to quantify calcification in vascular tissues using high resolution three-dimensional (3D) reconstructions of aortae derived from the well-established Ecto-nucleotide pyrophosphatase/phosphodiesterase-1 knockout $\left(\right.$ Enpp $\left.^{-/}\right)$mouse model of medial aortic calcification. A dual-contrast method was employed for specimen preparation and the application of corn oil as a submersion medium for the samples during scanning, which allowed the definition and quantification of soft tissue. 3D $\mu \mathrm{CT}$ was utilised to produce reconstructions of calcified and non-calcified aortae. A highly accurate quantification of a standardized region of calcium deposition was undertaken on these reconstructions. An excellent correlation between images obtained from $\mu \mathrm{CT}$ and those obtained with Alizarin red staining, of whole aortae for calcium deposition, was observed. This imaging protocol provides a powerful tool for studying the development of aortic calcification and potential therapeutic approaches for clinical intervention.
\end{abstract}

\section{Introduction}

Vascular calcification is a marker of increased cardiovascular risk in ageing and in a number of diseases including diabetes, atherosclerosis and chronic kidney disease (CKD) $(1,2)$. Although condition-specific factors are likely to drive the

Correspondence to: Dr V.E. MacRae, The Roslin Institute and Royal (Dick) School of Veterinary Studies, University of Edinburgh, Easter Bush, Roslin, Midlothian EH25 9RG, Scotland, UK

E-mail: vicky.macrae@roslin.ed.ac.uk

Key words: aorta, calcification, micro-computed tomography, quantification calcification process, the etiology of mineral accumulation within the vasculature resembles that of bone formation (2). A number of studies have reported that vascular smooth muscle cells (VSMCs), the predominant cell type involved in vascular calcification, can undergo a phenotypic transition to osteoblastic, chondrocytic and osteocytic cells in a calcified environment (3-5). Furthermore, it has been demonstrated that phosphate accelerates this phenotypic trans-differentiation, evident in the loss of characteristic smooth muscle markers and the development of osteoblastic features, such as the expression of tissue-nonspecific alkaline phosphatase, $\mathrm{P}_{\mathrm{i}} \mathrm{T}-1$, osteocalcin and osteopontin, and osteocyte markers including sclerostin and E11 $(3,4)$. Vascular calcification also involves the reciprocal loss of recognised calcification suppressors, such as inorganic pyrophosphate $\left(\mathrm{PP}_{\mathrm{i}}\right), \mathrm{MGP}$ and fetuin A (6-8).

To gain a better understanding of the mechanisms that underpin aortic calcification, rodent models of intimal and medial aortic calcification were utilised. However, the smallscale vascular structures in rodents require labor-intensive two-dimensional (2D) histomorphometric techniques in order to visualise regions of calcium and phosphate deposition using Alizarin red and von Kossa staining, respectively, with the extent of calcification often not being fully identified. Furthermore, use of the established technique of acid leaching to quantify total aortic calcium deposition in rodent tissue is of limited sensitivity and does not allow the visualisation of specific sites of calcification.

Recent reports have demonstrated the feasibility of detecting aortic calcification by micro-computed tomography ( $\mu \mathrm{CT}$ ) imaging (9-11). However, these studies examined calcified tissues without the assessment of soft tissues. In the present study, we developed a novel $\mu \mathrm{CT}$ scanning protocol to produce high resolution three-dimensional (3D) reconstructions of aortae derived from the well-established Ecto-nucleotide pyrophosphatase/phosphodiesterase-1 knockout $\left(\right.$ Enppl $\left.^{-/}\right)$mouse model of medial aortic calcification $(8,9,12)$. To the best of our knowledge, this is the first study which enables soft tissue definition and quantification, producing the first visualisation of calcified aortae. This new method is likely to advance the study of the progression of aortic calcification and potential therapeutic strategies for clinical intervention. 


\section{Materials and methods}

Enppl ${ }^{-/}$mice. Enpp1 $1^{-/}$mice were generated and characterised as previously described (12). Genotyping was performed by a commercial genotyping service (Genetyper, New York, NY, USA) using genomic DNA isolated from ear clips. All animal experiments were approved by The Roslin Institute's Animal Users Committee and the animals were maintained in accordance with the Home Office guidelines for the care and use of laboratory animals.

Tissue preparation. Aortae were dissected from 22-week-old Enpp $^{-/-}$and wild-type (WT) mice. Tissues were fixed in $10 \%$ neutral-buffered formalin for $48 \mathrm{~h}$ before being transferred to $70 \%$ ethanol. Prior to scanning, aortae were immersed, for a minimum time of $10 \mathrm{~min}$, in a macro-molecular iopamidolbased contrast agent (Niopam300; Brako UK Ltd., High Wycombe, Buckinghamshire, UK) diluted at 1:4 in water. To allow tissue differentiation, aortic lumina were filled with corn oil and the aortae were submersed in oil for the duration of the scan (Fig. 1).

Tissue imaging. Tissues were imaged using a Skyscan 1172 X-ray Microtomograph (Aartselaar, Belgium). Sequential high-resolution scans were obtained using a rotation step of $0.3^{\circ}$, with an average of 3 frames at each step, applying a $0.5 \mathrm{~mm}$ aluminium filter, with an X-ray source set at $60 \mathrm{kV}$ and $167 \mu \mathrm{A}$, and with an isotropic voxel size of $10 \mu \mathrm{m}$. Total scantime was $\sim 50 \mathrm{~min}$ per sample. The scans were reconstructed using NRecon (Skyscan, Kontich, Belgium). Noise, in the reconstructed images, was reduced by applying a median filter (radius, 1). The aortic arch, 400 slices (4 mm) under the subclavian artery, was selected as the region of interest. Soft and calcified tissue was identified by thresholding, using CTAn software (Skyscan). Thresholding permits the detection of differences in tissue density and thus enables the identification of areas of calcification within the soft tissue. The optimal threshold for each tissue type was determined from the image histograms (Fig. 2). Quantification was performed by calculating the total tissue volume within the specific threshold of the area of interest.

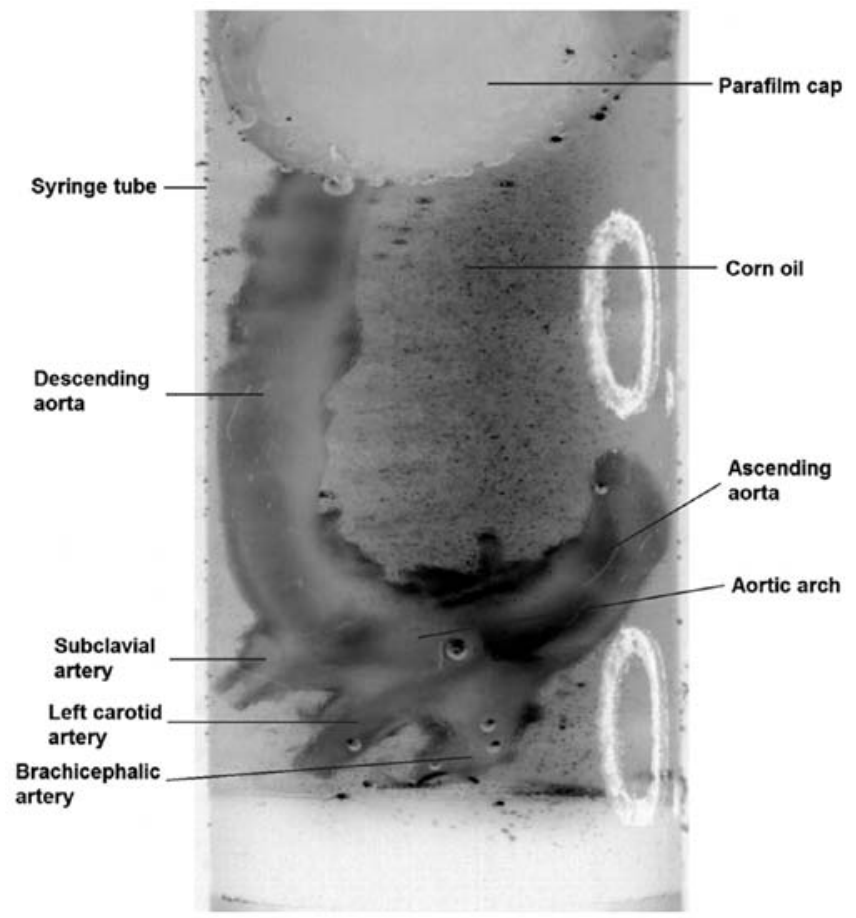

Figure 1. Sample preparation for micro-computed tomography $(\mu \mathrm{CT})$ scanner. The aorta is placed upside down inside a cut tube of a $1 \mathrm{ml}$ syringe. Movement of the aorta is restricted by positioning a piece of parafilm at the top of the aorta.

Calcification was expressed as the ratio of calcified volume to total volume.

Tissue staining for calcium deposition. After scanning, aortae were washed in PBS and stained with $2 \%$ Alizarin red ( $\mathrm{pH} 4.2)$ for $5 \mathrm{~min}$ at room temperature and rinsed repeatedly with distilled water. Images of whole aortae were captured with a low magnification microscope (magnification, x6; Leica MZ6 dissection microscope) in a darkened background.

Statistical analysis. Data are presented as means \pm SD. $\mathrm{P}<0.05$ indicates statistically significant difference as determined by Student's t-test analysis.
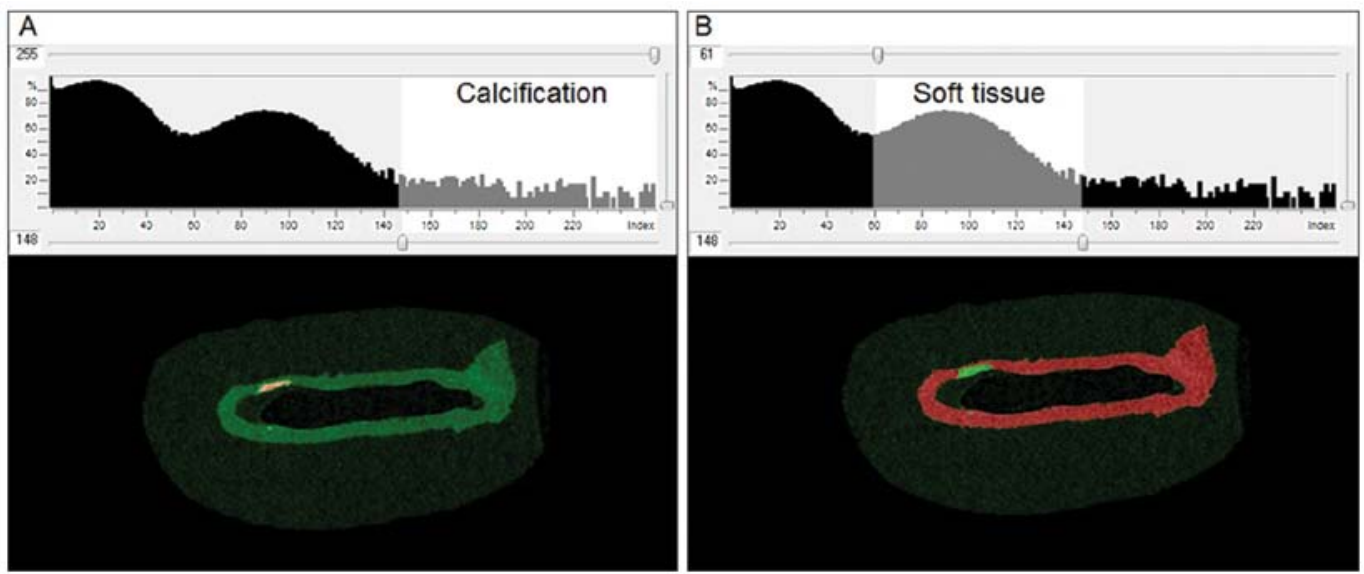

Figure 2. The selection of specific threshold values allowed the differentiation between (A) calcified tissue (values of 148-255) and (B) soft tissue (values of 61-148). Lower panels show a cross section of the aorta with red coloration indicating the selected tissue in that threshold and green coloration showing unselected tissue. 

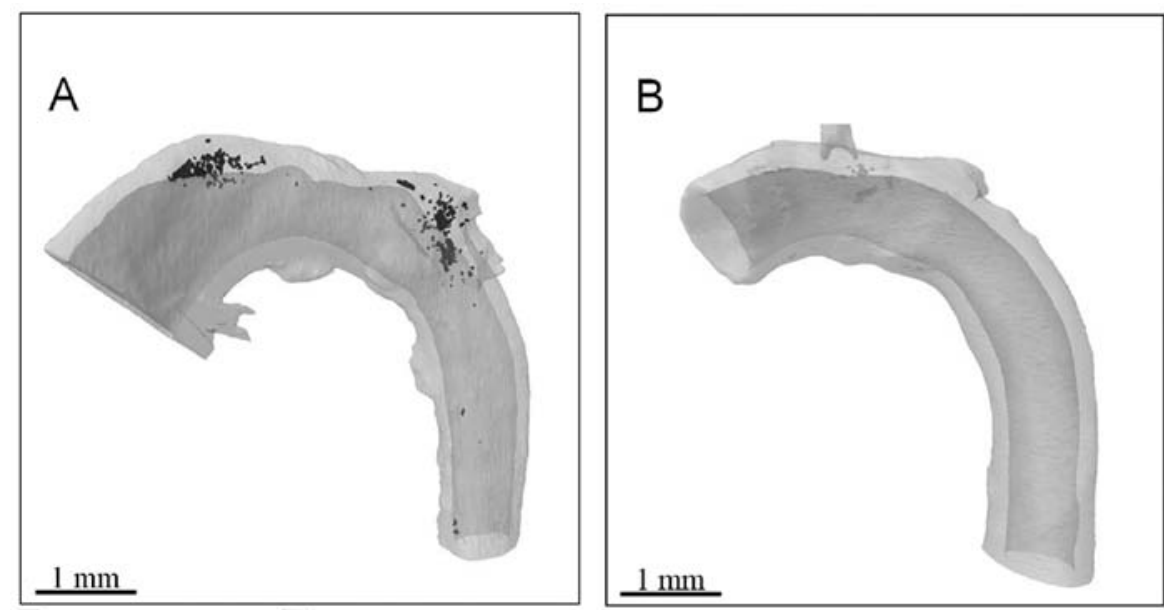

Figure 3. Three-dimensional volumetric reconstructions of (A) calcified Enpp $1^{-/}$and (B) non-calcified wild-type aorta at 22 weeks of age. Calcification is indicated by black coloring.
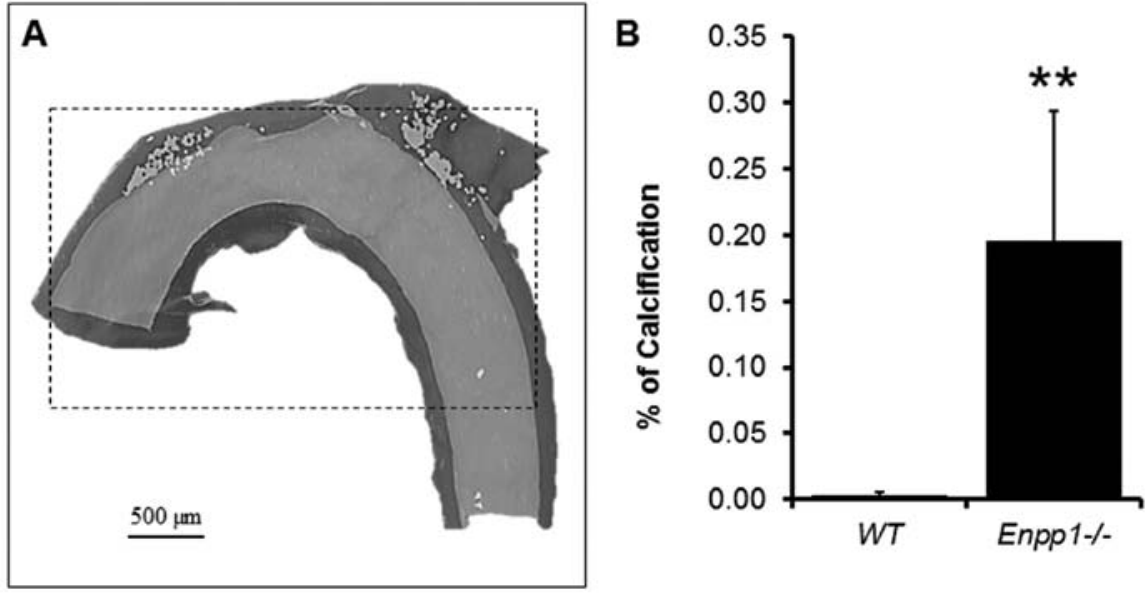

Figure 4. Quantification of calcium deposition. (A) A standardised region of calcium deposition (400 slices from the subclavian artery) was selected (B) Quantification of this region revealed a significant increase in calcium deposition in Enpp $1^{-1-}$ compared to the wild-type control aorta. Results are presented as mean \pm standard deviation, $\mathrm{n}=3{ }^{* * *} \mathrm{P}<0.01$.
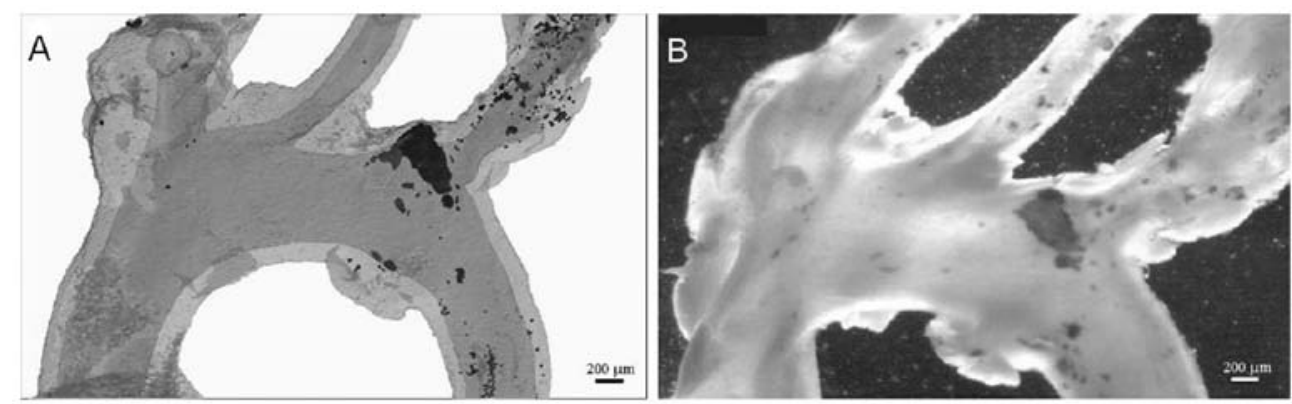

Figure 5. (A) Reconstructed micro-computed tomography $(\mu \mathrm{CT})$ image and (B) Alizarin red staining of the same aortic arch in an Enpp $1^{-\digamma}$ mouse.

\section{Results}

This study used calcified Enpp $1^{-/}$mouse aortae to demonstrate a novel $\mu \mathrm{CT}$ imaging protocol. A dual-contrast method was employed for specimen preparation combined with the use of corn oil during sample scanning (Fig. 1). While $\mu \mathrm{CT}$ has previously been utilised to image aortic calcification ex vivo,
(9-11), those studies detected calcified tissue in the absence of soft tissue assessment. Therefore, the 3D nature of $\mu \mathrm{CT}$ scanning has yet to be employed to fully exploit the potential for volumetric visualisation and assessment of multifocal regions of calcium deposition. The corn oil offered a different density to water, allowing a clearer definition of the soft tissue. It was therefore possible in the present study to reconstruct calcified 
(Fig. 3A) and non-calcified (Fig. 3B) aortae. A significant advantage of $3 \mathrm{D}$ reconstructions is that the rotation of an image around any axis is possible, without the limitations associated with interpretation on a single plane.

A highly accurate quantification of a standardized region of calcium deposition (400 slices from the subclavian artery) was undertaken on the reconstructions (Fig. 4). In the three samples measured, it was possible to quantify the exact amount of volume corresponding to the mineral threshold compared to the soft tissue of the standardised area selected. For comparison purposes, the same aortae were stained with Alizarin red, which allowed visualisation of the areas of calcification within the soft tissue. An excellent visual correlation was observed between stained aortae and the corresponding $\mu \mathrm{CT}$ reconstructions (Fig. 5), indicating that the selected thresholds corresponded to the mineral and soft tissue, respectively.

\section{Discussion}

Results of the present study demonstrate the suitability of $\mu \mathrm{CT}$ for the assessment of calcification in isolated arteries. We report a novel method for specimen preparation and soft tissue definition that produces high-resolution 3D images and demonstrates the first visualisation of calcified aortae. Scanning of the sample, submerged in corn oil, provides anatomical information on the size and distribution of calcified regions within the soft tissue, as well as reproducible quantification of both the calcified area and the residual soft tissue. Even though in the present study this was undertaken in mouse aortae, this technique would be equally suitable for analysis of blood vessels from other species, including small human vessels. The methodology described in the present study represents considerable improvement over traditional histological approaches, chemical assays and previously reported $\mu \mathrm{CT}$ imaging protocols for the analysis of calcium deposition in arteries (9-11).

The key innovation of this study is the use of a fluid with a markedly different density to water during the $\mu \mathrm{CT}$ scan. Soft tissue, which is comprised predominantly of water and collagen, has previously been difficult to visualise with $\mu \mathrm{CT}$ scanning of aortic calcification, due to the comparable densities of water and soft tissue. The inability to assess the calcification volume with respect to the whole tissue has therefore limited the application of $\mu \mathrm{CT}$ in the vascular calcification field to date. The capacity to accurately quantify calcification and tissue volume is therefore a major strength of the technique described in the present study. Additional benefits of using the oil-immersion method include the prevention of i) drying and shrinkage of the tissue and ii) leaching of the dual-contrast agent during scanning. The disadvantages of the technique are that it can only be carried out ex vivo and that connective tissues attached to the aortae are indistinguishable from the aorta itself. It is therefore extremely important to clean the aortae of such tissues after dissection. Even considering these minor disadvantages, compared to histological approaches, this method of quantification is considerably more representative of the total calcification burden in an artery, and is not subjected to the bias and error incurred by selecting areas of analysis either randomly along the profile of the vessel, or at the site of maximum calcification. This is emphasised in the current study, with 3D reconstructions indicating a notable variation in calcium deposition over relatively short lengths of the vessel (Fig. 3).

Imaging by $\mu \mathrm{CT}$ is relatively convenient, fast and high throughput. Therefore, the method reported herein provides the means to allow the highly accurate $3 \mathrm{D}$ evaluation of morphology to become a routine component of ex vivo vascular calcification studies. 3D optical imaging modalities have also been recently employed to spatially and temporally resolve and quantify dynamic pro-calcification molecular mechanisms in mouse aortae in vivo (13). Thus, these 3D imaging systems provide powerful tools to study the progression of aortic calcification and potential therapeutic strategies for clinical intervention.

\section{Acknowledgements}

This study was supported by an Institute Strategic Programme Grant and Institute Career Path Fellowship funding from the Biotechnology and Biological Sciences Research Council (BBSRC).

\section{References}

1. Mackenzie NC and MacRae VE: The role of cellular senescence during vascular calcification: a key paradigm in aging research. Curr Aging Sci 4: 128-136, 2011.

2. Zhu D, Mackenzie NC, Farquharson $\mathrm{C}$ and Macrae VE: Mechanisms and clinical consequences of vascular calcification. Front Endocrinol (Lausanne) 3: 95, 2012.

3. Speer MY, Yang HY, Brabb T, et al: Smooth muscle cells give rise to osteochondrogenic precursors and chondrocytes in calcifying arteries. Circ Res 104: 733-741, 2009.

4. Zhu D, Mackenzie NC, Millán JL, Farquharson C and MacRae VE: The appearance and modulation of osteocyte marker expression during calcification of vascular smooth muscle cells. PLoS One 6: e19595, 2011.

5. Zhu D, Mackenzie NC, Millan JL, Farquharson C and MacRae VE: A protective role for FGF-23 in local defence against disrupted arterial wall integrity? Mol Cell Endocrinol 372: 1-11, 2013.

6. Murshed M, Harmey D, Millan JL, McKee MD and Karsenty G: Unique coexpression in osteoblasts of broadly expressed genes accounts for the spatial restriction of ECM mineralization to bone. Genes Dev 19: 1093-1104, 2005.

7. Rutsch F, Ruf N, Vaingankar S, et al: Mutations in ENPP1 are associated with 'idiopathic' infantile arterial calcification. Nat Genet 34: 379-381, 2003.

8. Mackenzie NC, Huesa C, Rutsch F and MacRae VE: New insights into NPP1 function: lessons from clinical and animal studies. Bone 51: 961-968, 2012.

9. Postnov AA, D'Haese PC, Neven E, De Clerck NM and Persy VP: Possibilities and limits of X-ray microtomography for in vivo and ex vivo detection of vascular calcifications. Int J Cardiovasc Imaging 25: 615-624, 2009.

10. Persy V, Postnov A, Neven E, et al: High-resolution X-ray microtomography is a sensitive method to detect vascular calcification in living rats with chronic renal failure. Arterioscler Thromb Vasc Biol 26: 2110-2116, 2006.

11. Awan Z, Denis M, Bailey D, et al: The LDLR deficient mouse as a model for aortic calcification and quantification by microcomputed tomography. Atherosclerosis 219: 455-462, 2011.

12. Mackenzie NC, Zhu D, Milne EM, et al: Altered bone development and an increase in FGF-23 expression in Enpp1(-/-) mice. PLoS One 7: e32177, 2012.

13. Aikawa E, Nahrendorf M, Figueiredo JL, et al: Osteogenesis associates with inflammation in early-stage atherosclerosis evaluated by molecular imaging in vivo. Circulation 116: 2841-2850, 2007. 